\title{
Description of cause of serious illness and outcome in patients identified using ETAT guidelines in urban Malawi
}

\author{
M A Robertson, E M Molyneux
}

\begin{abstract}
Aims-To evaluate the performance of guidelines for emergency triage and treatment (ETAT) of children presenting to hospitals in the developing world. Part of the study was concerned with the delivery of emergency treatment to the sickest group of patients, characterisation of their illness, and outcome.

Methods and results-A total of 236 children were admitted during the study period, 27 of whom died. The three main causes of death were malaria or malaria related illness $(n=7)$, pneumonia $(n=6)$, and malnutrition $(n=11)$. Forty seven children were categorised as needing emergency treatment. Thirty one had no treatment, and eight died; 16 received one or more recommended treatments, of whom five died. The main limitations to delivery of immediate care were the lack of staff in the department and lack of rapidly available blood.

(Arch Dis Child 2001;85:214-217)
\end{abstract}

Keywords: emergency care; developing world

The World Health Organisation (WHO) has developed guidelines for emergency triage, assessment, and treatment of sick children presenting to hospitals in the developing world (ETAT). ${ }^{1}$ This is part of the integrated management of childhood illness. ${ }^{2}$ The validation study to pretest ETAT in Malawi, where the performance of the triage guidelines was discussed is described elsewhere. ${ }^{3}$

This paper describes the cause of serious illness in children as identified by the ETAT guidelines, their admission diagnoses, emergency treatment delivered at the time of triage, disposal (admission, discharge, transfer), and final outcome (survival, death). Limitations to the delivery of care or the processing of patients, by both nurses and clinical officers, were also noted.

Department of

Paediatrics, Queen Elizabeth Central Hospital, Blantyre, Malawi

M A Robertson

E M Molyneux

Correspondence to: Prof. Molyneux

emolyeux@malawi.net

Accepted 2 May 2001

\section{Methods}

The Queen Elizabeth Central Hospital in Blantyre is the teaching and referral hospital for the southern region of Malawi. It serves both adult and paediatric populations. The number of children seen per annum averages 100000 . About 10000 of these are admitted and inpatient mortality is approximately $10 \%$. A departmental audit from January to June 1997 recorded the main causes of inpatient paediatric death as malaria with or without anaemia, pneumonia, septicaemia, gastroenteritis, AIDS, malnutrition, acute respiratory infection, dehydration, and tuberculosis. Some of these diagnoses overlapped. ${ }^{4}$ The national under $5 \mathrm{~s}$ mortality rate is 189 per 1000 live births. Thirty per cent of all children are underweight, $49 \%$ are stunted, and $6 \%$ are severely wasted. ${ }^{5}$ In a recent study HIV prevalence was found to be $18 \%$ of all paediatric admissions to the Queen Elizabeth Central Hospital. ${ }^{6}$

The staffing of the department, study protocol, and training programme are described elsewhere. ${ }^{3}$

Appendix 1 illustrates emergency triage signs used in the study and the treatment recommended when emergency signs are present. The emergency treatments were developed for use in a poorly resourced setting, with a very limited pharmacopoeia. Treatments relate to the immediate management of conditions where morbidity and mortality were felt to be preventable by simple interventions. Cardiopulmonary resuscitation, with or without intubation, was not included, because of the limited availability of intensive care facilities and the known poor outcome in children with cardiac arrest that is not witnessed. ${ }^{7-21}$

During staff training bag and mask ventilation and insertion of intraosseous needles was demonstrated using simulation techniques. Considerable time was given to teaching the calculation of fluid and drug doses for children of varying weights. Laminated charts displaying these were created for use in the emergency treatment room.

All children who were assessed as needing emergency treatment were allocated to priority one (P1), and transferred to the emergency room. A trained paediatric emergency doctor supervised treatment, and treatment decisions were recorded on the assessment form.

Children who required an urgent medical assessment were assigned priority two (P2), and these patients were brought to the front of the queue waiting to see the medical officer. Priority three (P3) patients were those who could wait safely and were seen in order of attendance.

The initial 700 children triaged were not tracked through the department to final outcome because of staffing difficulties. The remaining 1581 cases were followed through to a decision to admit or discharge, and the clinical officer's admission diagnosis recorded. At the end of the study period the outcome of triaged children who had been admitted was determined as far as possible from available hospital records. 

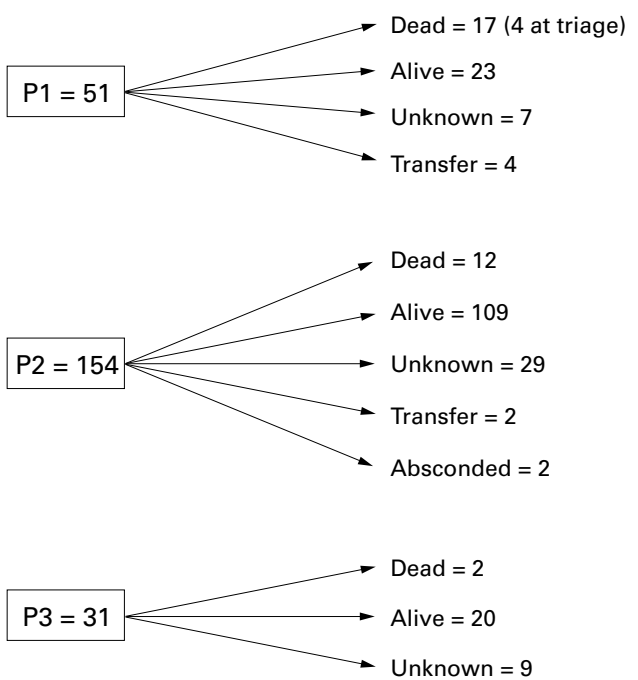

Figure 1 Final outcome of patients admitted $(n=236)$.

Table 1 Diagnosis on admission; association with priority

\begin{tabular}{llllr}
\hline Diagnosis & Total number & $P 1$ & $P 2$ & $P 3$ \\
\hline Malaria & 69 & 7 & 45 & 16 \\
Pneumonia & 26 & 6 & 17 & 3 \\
Gastroenteritis/dehydration & 23 & 3 & 16 & 4 \\
Anaemia/malaria & 21 & 4 & 14 & 3 \\
Kwashiorkor & 19 & 2 & 17 & 1 \\
Anaemia & 11 & 2 & 7 & 2 \\
Other & 9 & 2 & 7 & 0 \\
Sepsis & 7 & 1 & 6 & 0 \\
Cerebral malaria & 7 & 6 & 1 & 0 \\
Cholera & 6 & 4 & 2 & 0 \\
Marasmus & 5 & 1 & 4 & 0 \\
Oedema/other & 5 & 1 & 4 & 0 \\
Respiratory/other & 4 & 1 & 3 & 0 \\
Dead at triage & 4 & 4 & 0 & 0 \\
Unknown & 4 & 2 & 1 & 1 \\
Meningitis & 3 & 0 & 3 & 0 \\
Fits & 3 & 2 & 1 & 0 \\
Burns & 2 & 2 & 0 & 0 \\
Malaria/pneumonia & 2 & 0 & 1 & 1 \\
Malaria/meningitis & 2 & 1 & 1 & 0 \\
Anaemia/kwashiorkor & 2 & 0 & 2 & 0 \\
Kwashiorkor/pneumonia & 2 & 0 & 2 & 0 \\
\hline
\end{tabular}

Table 2 Diagnosis related to priority in patients dying within 24 hours of admission

\begin{tabular}{llll}
\hline Diagnosis & $P 1$ & $P 2$ & $P 3$ \\
\hline Malaria and/or anaemia & 6 & 1 \\
Pneumonia & 1 & 2 & \\
Malnutrition & 1 & 1 & \\
Unknown & & 1
\end{tabular}

\section{Results}

A total of 2281 children were triaged during the study period. Of these, 1581 were followed through to admission or discharge. For all admitted children the final outcome was retrieved from inpatient records. Thirty nine per cent $(n=884)$ of children were aged 1 year or younger. Forty six per cent $(n=1042)$ were over 1 year but under 5 years, and the remaining 15 per cent $(n=350)$ were 5 years or over.

Of the 1581 children triaged and followed up, 236 (15\%) were admitted; fig 1 shows the final outcome for each triage group. Table 1 shows admission diagnoses, with numbers of patients admitted in each priority group. Over $60 \%(n=19)$ of the children in P3 who were admitted had malaria or a malaria related illness.
Table 3 Diagnosis related to priority in patients dying after first 24 hours

\begin{tabular}{llll}
\hline Diagnosis & $P 1$ & $P 2$ & $P 3$ \\
\hline Malnutrition & 3 & 6 & 1 \\
Pneumonia & 1 & 1 & 1 \\
Unknown & 1 & & \\
\hline
\end{tabular}

Thirty one children died, four were found dead at triage, and 27 died after admission. The 27 post-admission deaths represented $12 \%$ of all admissions; this is in keeping with an overall inpatient mortality of $10 \%{ }^{4}$

Tables 2 and 3 show the total number of deaths in the study period, early deaths being those occurring within 24 hours of admission. Malaria and anaemia were responsible for just over half of all early deaths, and for $75 \%$ of early deaths in the P1 group. No later deaths were attributed to these conditions.

Pneumonia caused nearly a quarter (six of 27) of all deaths. Eleven deaths (41\%) were related to malnutrition, although only one occurred within 24 hours of admission. The mean survival for those in the late death group was seven days (range 3-18 days).

All P1 patients were to be considered for the emergency treatments shown in Appendix 1. Table 4 shows interventions received by these children and their final outcome. Thirty one patients $(66 \%)$ received no treatment, three died early, and five late. Sixteen patients received treatment, of whom five died within 24 hours, but there were no late deaths. Table 5 shows all patients in the P1 category that died, with their diagnosis and any emergency treatment provided.

\section{Discussion}

Data were collected to describe the cause of serious illness in children identified by ETAT guidelines. The three main causes of death in the study period were malaria/anaemia, pneumonia, and malnutrition.

The administration of emergency treatment in the under 5 s clinic was a new concept at the start of this study. The nursing staff see many children who are seriously ill. Previous practice had been to transfer such patients to the admissions ward as soon as possible, where most patients had a second and often prolonged wait for definitive treatment. The effect of early intervention on outcome for P1 patients was examined along with limitations in its delivery.

One third of P1 patients received emergency treatment (as advised by the ETAT protocol) during the study period, and mortality among these was slightly higher $(31 \%)$ than in those P1 children receiving no treatment (26\%). All deaths within 24 hours of admission of P1 patients occurred in the group who had received emergency treatment, and it is probable that these were recognised by the nursing staff to be in extremis on presentation. There were three early deaths among the patients who received no treatment, two children with anaemia/malaria and one whose diagnosis remained elusive (possibly organophosphate poisoning). 
Table 4 Emergency treatment and outcome for P1 group

\begin{tabular}{lllllll}
\hline Treatment & Total & Alive & Transfer & Died early & Died late & Unknown \\
\hline None & 31 & 15 & 2 & 3 & 5 & 6 \\
Oxygen & 4 & 0 & 0 & 4 & 0 & 0 \\
IV fluid & 10 & 3 & 3 & 1 & 0 & 3 \\
Anticonvulsant & 1 & 1 & 0 & 0 & 0 & 0 \\
BM test & 2 & 1 & 0 & 0 & 0 & 1 \\
\hline
\end{tabular}

Table 5 Early and late deaths with interventions received in P1 patients

\begin{tabular}{lll}
\hline Disease & Early deaths & Late deaths \\
\hline Anaemia/malaria & 6 & 0 \\
Rx: None & 2 & \\
IV & 1 & \\
$\mathrm{O}_{2}$ & 3 & 1 \\
Pneumonia & 1 & 1 \\
Rx: None & 0 & 0 \\
$\mathrm{O}_{2}$ & 1 & 3 \\
Malnutrition & 0 & 3 \\
Rx: None & & 1 \\
Unknown & 1 & 1 \\
$\quad$ Rx: None & 1 & \\
\hline
\end{tabular}

Although actual numbers are small $(n=4)$, $100 \%$ of children receiving supplemental oxygen were dead within 24 hours of admission. This reflects the severity of illness in these patients and the underlying diagnosis of malaria/anaemia in the majority. One of the 10 children receiving intravenous fluid died early; this was a child with anaemia/malaria.

Malaria is endemic in Malawi and is the major cause of acute anaemia in children. The two diagnoses were not considered separately. Parasitaemia was found in some other admitted children, but was thought not to be the main reason for admission, nor to contribute significantly to outcome.

Anaemia/malaria represented 54\% ( $\mathrm{n}=7)$ of all early deaths (both P1 and P2 patients). In P1 patients with anaemia/malaria, three received supplemental oxygen, one received intravenous fluid, and two received no treatment.

Two of the three children who were given oxygen died before their packed cell volume (PCV) could be estimated, and the third child who received supplemental oxygen had a PCV of $9 \%(\mathrm{Hb} 30 \mathrm{~g} / \mathrm{l})$. The child given intravenous fluids had a PCV of $20 \%$, and the two receiving no treatment had PCVs of $15 \%$ and $34 \%$. This last child died of cerebral malaria. The P2 child died before her PCV could be established, having arrived too late for successful resuscitation. The treatment most likely to offer survival benefit for these patients is a blood transfusion. Non-relative donors are few and family members are usually recruited to donate blood. It may take the family time to identify a donor, especially if they come from far away. Many test positive for HIV, or have otherwise incompatible blood. In addition the transfusion laboratory is under staffed, and out of hours donor screening was unavailable. Therefore blood can be difficult to obtain rapidly and a number of severely anaemic children die as a result.

The main limitation to implementation of emergency treatment was insufficient staffing in the department and no appropriate emergency treatment for the child with severe anaemia/malaria.
Nursing staff in the under 5 s clinic highlighted another influence on outcome. The introduction of triage into the clinic meant that the sickest patients were being treated and transferred to the admissions unit earlier in the day. This change had a knock on effect. In the hospital teams responsible for inpatient care do their ward rounds in the morning. This practice was ongoing during the study, so patients transferred to the admissions unit could still wait to be seen by the admitting doctor. The inpatient teams had to restructure their working practice to care effectively for these admissions.

The data on patients in this study are not complete, but there had, in the previous two years, been 10 deaths a month in the clinic from December to June (Molyneux EM, personal communication, under 5 s clinic figures for 1996, 1997). This has reduced to five. The clinic staff felt that patient flow was more efficient and that the sickest children were waiting far less time in the under $5 \mathrm{~s}$ clinic before being admitted.

Any reorganisation in one sector effects the rest of the unit. Improved triage highlighted the need for more emergency treatments to be available in the clinic and for the staff to implement them. Earlier referral to the inpatient team required a change in practice. The on call junior doctor received the admissions sent to the wards, while the rest of the team did the ward round.

Since completing this study the department has been altered structurally and working practices have changed. An improved and enlarged physical setting has made it possible to have a separate resuscitation room to which priority one patients are taken straight from the triage station. Staff are able to initiate resuscitation. This has reduced delays and built up the confidence and morale of staff. We are monitoring the outcome of children admitted through this room. In the first 100 patients the mortality has been $25 \%$. This compares favourably with a mortality of $35 \%$ of priority one patients admitted during the study. The employment of an extra clinical officer has meant that priority two patients can be seen "at the front of the queue" without causing irritation and delay to the priority three group.

Malnutrition per se is not seen as an emergency as no immediate resuscitation will alter outcome. Severe malnutrition requires careful, long term inpatient management. It is triaged as priority two and seen by the admission team.

The admission team has moved into the renovated building, reducing further delays. Blood films for malaria parasites, a haematocrit, and blood glucose are carried out on finger prick blood samples of all children seen in the resuscitation and admission rooms, hastening and improving management.

We would like to thank the Department of Child and Adolescent Health and Development (World Health Organisation) for supporting this study: Dr Vicky Lavy and Susan Champion for help in training, and testing the use of ETAT, and all the clinic staff involved in the care of children. 
Any sign positive

$\frac{\text { Airway and }}{\text { breathing }}$

A \& B

Circulation

$\mathrm{C}$

Coma

Convulsions

Confusion

$\mathrm{Cm} \mathrm{Cn} \mathrm{Cf}$

Dehydration

(child has vomiting

or diarrhoea)

D

\section{PRIORITY SIGNS}

Severe wasting

Child under 2 months of age

Irritable or restless

Pallor

Major burn

A child with any priority sign needs urgent assessment

Figure A1 Emergency signs.

\section{Appendix 1: Emergency signs}

Figure A1 presents the system of priority signs.

1 Gove S, Tamburlini G, Molyneux E, et al (for the WHO IMIC Referral Care Project). The development and technical basis of simplified guidelines for emergency triage assessment and treatment in developing countries. Arch Dis Child 1999;81:473-7.

2 Gove S (for the WHO working Group on Integrated Management of Childhood Illness). Integrated management of childment of Childhood Illness). Integrated management of childhood illness by outpatient health workers: technical basis and

3 Robertson MA, Molyneux E. Triage in the developing world - can it be done? Arch Dis Child 2001;85:208-13.

4 Molyneux E, Broadhead R. Unpublished audits for paediatric department at Queen Elizabeth Central Hospital, Blantyre, 1997. The Malawi Demographic and Health Survey 2000. National Statistical Office, PO Box 333, Zomba, Malawi, 2000.

6 Gladstone MJ, Callaghan M, Rogerson SJ, et al. HIV prevalence in paediatric admissions in Malawi. Arch Dis Child 2001;84(suppl):A43.

7 Kuisma M, Suomien P, Korpela R. Paediatric out-ofhospital cardiac arrests-epidemiology and outcome. Resuscitation 1995;30:141-50.

8 Hassan TB. Use and effect of paediatric advanced life support skills for paediatric arrest in the A\&E department. $\mathcal{F}$ Accid Emerg Med 1997;14:357-62.

9 Suominen P, Rasanen J, Kivioja A. Efficacy of cardiopulmonary resuscitation in pulseless paediatric trauma pamonary resuscitation in pulseless
tients. Resuscitation $1998 ; 36: 9-13$.
Oedema both feet

Lethargy

Any respiratory distress

Urgent referral from another health facility
Manage airway

Give oxygen

Remove any foreign body

Stop bleeding

Give oxygen

Give intravenous

fluids $20 \mathrm{ml} / \mathrm{kg}$

Manage airway

Give oxygen

Rectal diazepam

Give i.v. dextrose $10 \%$

Position child

Give i.v. or nasogastric

fluids
10 Erlich R, Emmet SM, Rodriguez-Torres R. Paediatric cardiac resuscitation team: a six-year study. $\mathcal{F}$ Pediatr 1974; 84:152-5.

11 Friesen RM, Duncan P, Tweed WA, Bristow G. Appraisal of pediatric cardiopulmonary resuscitation. Can Med Assoc 7 1982;126:1055-8

12 Eisenberg M, Bergner L, Hallstrom A. Epidemiology of cardiac arrests and resuscitation in children. Ann Emerg Med 1983;12:672-4.

13 Lewis JK, Minter MG, Eshelman SJ, Witte MK. Outcome of paediatric resuscitation. Ann Emerg Med 1983;12:297-9.

14 Torphy DE, Minter MG, Thompson BM. Cardiorespiratory arrest and resuscitation of children. Am $\mathcal{f}$ Dis Child 1984;138:1099-102

15 O'Rourke PP. Out of hospital cardiac arrest in paediatric patients: outcome [abstract]. Crit Care Med 1984;12:283.

16 Barzilay Z, Somekh E, Sagy M, Boichis H. Pediatric cardiopulmonary resuscitation outcome. 7 Med 1988;19:229-41.

17 Thompson JE, Bonner B, Lower GM. Pediatric cardiopulmonary arrests in rural populations. Pediatrics 1990;86:302-6.

18 Innes PA, Summers CA, Boyd IM, Molyneux EM. Audit of paediatric cardiopulmonary resuscitation. Arch Dis Child 1993;68:487-91.

19 O'Rourke PP. Outcome of children who are apneic and pulseless in the emergency room. Crit Care Med 1986;14: 466-8.

20 Orlowski JP. The effectiveness of pediatric cardiopulmonary resuscitation. Am f Dis Child 1984;138:1097.

21 Paediatric Life Support Working Party for the European Resuscitation Council. Guidelines for paediatric life support. BMF 1994;308:1349-55. 